\title{
Development and Evaluation of a Bayesian Risk Stratification Method for Major Amputations in Patients with Diabetic Foot Ulcers
}

\author{
Jens HÜSERS ${ }^{\mathrm{a}, 1}$, Guido HAFER ${ }^{\mathrm{b}}$, Jan HEGGEMANN ${ }^{\mathrm{b}}$, Stefan WIEMEYER ${ }^{\mathrm{b}}$, \\ Swen Malte $\mathrm{JOHN}^{\mathrm{c}}$ and Ursula HÜBNER ${ }^{\mathrm{a}}$ \\ ${ }^{a}$ Health Informatics Research Group, Osnabrück University AS, Germany \\ ${ }^{b}$ Christliches Klinikum Melle, Niels Stensen Kliniken, Germany \\ ${ }^{c}$ Department Dermatology, Environmental Medicine, Health Theory, \\ University of Osnabrück, Germany
}

\begin{abstract}
The diabetic foot ulcer, which 2\% - 6\% of diabetes patients experience, is a severe health threat. It is closely linked to the risk of lower extremity amputation (LEA). When a DFU is present, the chief imperative is to initiate tertiary preventive actions to avoid amputation. In this light, clinical decision support systems (CDSS) can guide clinicians to identify DFU patients early. In this study, the PEDIS classification and a Bayesian logistic regression model are utilised to develop and evaluate a decision method for patient stratification. Therefore, we conducted a Bayesian cutpoint analysis. The CDSS revealed an optimal cutpoint for the amputation risk of 0.28 . Sensitivity and specificity were 0.83 and 0.66 . These results show that although the specificity is low, the decision method includes most actual patients at risk, which is a desirable feature in monitoring patients at risk for major amputation. This study shows that the PEDIS classification promises to provide a valid basis for a DFU risk stratification in CDSS.
\end{abstract}

Keywords: Clinical Decision Support System, Logistic Regression, Health Information Technology, Diabetic Foot Ulcer, Amputation, Bayesian statistics

\section{Introduction}

The diabetic foot ulcer (DFU), which $2 \%$ - $6 \%$ of diabetes patients experience, is a severe health threat that is characterized as a chronic lesion of the foot tissue, which is mainly caused by diabetes-related peripheral vascular impairment and neuropathic conditions [1]. The prevention of diabetes-related foot problems is the primary imperative, as they are linked with the risk of lower extremity amputation (LEA), which is associated with high economic and health burden [2]. This is especially true for amputations above the ankle, also defined as major amputations. When a DFU is present, it is desirable to identify major-amputation risk patients to initiate preventive actions early on.

${ }^{1}$ Corresponding Author, Jens Hüsers, Osnabrück University of AS, Health Informatics Research Group, PO Box 1940, 49009 Osnabrück, Germany; E-Mail: j.huesers@hs-osnabrueck.de. 
The PEDIS classification, which reliably describes DFU through five risk factors (Perfusion status, ulcer Extent and Depth, Infection status, and foot Sensation) [3,4], is an international standard for DFU classification consented by the International Working Group of the Diabetic Foot. It is the aim of this study to develop a model based stratification scheme that allows for the classification of patients with and without a risk for a major amputations using the PEDIS system.

Accordingly, our research questions concerned the development (first question) and the evaluation (second question): 1.) Which risk (probability) constitutes the optimal cutpoint for major-amputation risk stratification? 2.) What is the corresponding diagnostic accuracy in terms of AUC, sensitivity and specificity?

\section{Methods}

We conducted a prospective cohort study at the Wound Care Centre of Christliches Klinikum Melle in Germany, which started in June 2013 and ended in June 2019. All subjects were screened before inclusion: Diabetes mellitus (type I and type II) patients with a DFU were included; other wounds, e.g., venouse or arteric leg ulcers, were excluded. Then, eligible subjects underwent baseline assessment where age and gender were documented, as well as the ulcer classified according to the PEDIS system. Six months after baseline assessment, the major-amputation status, defined as amputation above the ankle was determined and recorded. This prospective data collection was repeated, when no major-amputation was conducted, and the treatment was continued for a patient.

We aimed to stratify patients for the target condition, i.e., major amputation. Therefore, we utilised a Bayesian logistic regression model [5], in which majoramputation served as the criterion, each of the five PEDIS risk factors as a predictor and gender as well as age as covariates. We selected a logistic regression model as it allowes meaningful interpretation of the model parameters, i.e., odds ratio and therefore provides transparent insights for clinicians. Furthermore, the bayesian approach allows the incorporation of prior knowledge. In this study the bayesian model made use of information from the multicentre EURODIALE study [6].

The Bayesian posterior distributions of the model coefficients were the starting point for the cutpoint analysis, which was based on maximising the Youden-Index [7]. To analyse the diagnostic validity of the cutpoint we computed the AUC as well as sensitivity and specificity and their $95 \%$ high-density intervals (HDI) to quantify uncertainty [8].

\section{Results}

The data used to fit the model was based on a sample of $237 \mathrm{DFU}$ patients. Of all patients, $12.2 \%(\mathrm{n}=29)$ underwent major-amputation procedure. The mean age of the overall sample was 65.9 years $( \pm 12.3)$. For the non-amputees and amputees, the average age was 65.6 years $( \pm 12.5)$ and 65.5 years $( \pm 12.4)$, respectively. The overall proportion of female and male patients was $16.5 \%(\mathrm{n}=39)$ and $83.5 \%(\mathrm{n}=198)$.

According to the Youden-Index, the posterior median estimate of the optimal cutpoint was 0.28 , which means that patients with a predicted amputation probability above this threshold are classified as risk patients. Quantifying the uncertainty associated 
with this estimate, the $95 \%$ HDI ranged from 0.16 to 0.41 . The posterior median of the sensitivity was $0.83(95 \%$ HDI $0.71-0.93)$, and that of the specificity was $0.66(95 \%$ HDI 0.53 - 0.77) (Figure 1).

Table 1. Optimal cutpoint, $\mathrm{AUC}$ and the screening test metrics with corresponding $95 \%$ highest density intervals.

\begin{tabular}{rcr}
\hline Metric & $\begin{array}{c}\text { Posterior } \\
\text { Median }\end{array}$ & 95\% HDI \\
\hline Optimal Cutpoint & 0.28 & {$[0.16-0.41]$} \\
AUC & 0.80 & {$[0.78-0.80]$} \\
\hline Sensitivity & 0.83 & {$[0.71-0.93]$} \\
Specificity & 0.66 & {$[0.53-0.77]$} \\
\hline
\end{tabular}

\section{Discussion}

In this study, we developed a PEDIS based method for binary risk stratification for DFU patients facing potential major-amputation. The optimal cutpoint for the predicted sixmonth amputation risk was determined by drawing on a Bayesian logistic regression model with an acceptable AUC value and its stratification performance was scrutinised using diagnostic metrics.
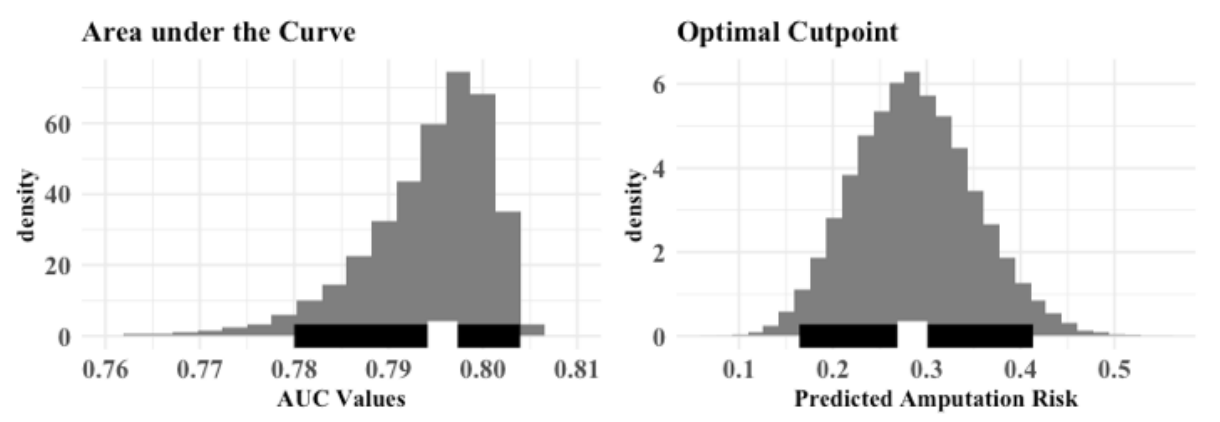

Figure 1. Histogram of posterior distribution of AUC (left) and cutpoint (right), horizontal black bar indicates range of $95 \%$ HDI and white box within the bar indicates the median. Left: AUC median $=0.80$ (95\% HDI 0.78 - 0.80) Right: Optimal cutpoint median $=0.28$ (95\% HDI 0.16 to 0.41$)$.

Using the Youden index for cutpoint determination, the optimal cutpoint was 0.28. Patients with higher predicted risks are considered as patients at risk. The associated HDI ranges from 0.16 to 0.41 ; thus, indicating some uncertainty about the optimal cutpoint.

The sensivity and specificity was 0.83 and 0.66 , respectively. These results reflect a typical situation where a tradeoff between sensitivity and specificity is present. This trade-offs have to be discussed critically: In this case, the rather low specificity is tolerable for the context of amputation because higher sensitivity is a desirable feature from the clinical perspective of monitoring these patients more closely: Through this test, a clinician can detect most of the actual risk patients at the expense of including non-risk patients in the risk strata.

As this method requires computational ressources, we recommend to implement it in routine record-keeping systems such as wound documentations as a clinical decision 
support tool. Thereby, the clinician receives valuable additional information about the risk status of a DFU patient when completing the PEDIS classification procedure.

A limitation of this study is its single-centre approach. Thus, this study may represent a specific subgroup seen in specialised, multidisciplinary wound care centres rather than patients that nurses and general practitioners see in daily routine care. However, by incorporating external scientific knowledge in the Bayesian model, we tried to mitigate this effect. Furthermore, more data is required. Again, the Bayesian approach allows an update of the model when further data is available.

In summary, this study leads to two main conclusions. First, Bayesian statistics is a valuable method for designing and evaluating clinical decision models. Second, given further validation through more data, the PEDIS classification promises to be a valid and clinically applicable method for risk stratification for patients with a DFU.

\section{Funding}

The state of Lower Saxony funded this work, project ROSE - The Learning Health Care System in the region Osnabrück and Emsland (ZN3103)

\section{References}

[1] Zhang P, Lu J, Jing Y, Tang S, Zhu D, Bi Y. Global epidemiology of diabetic foot ulceration: a systematic review and meta-analysis. Ann Med. 2017 Feb 17;49(2):106-16.

[2] Bakker K, Apelqvist J, Lipsky BA, Van Netten JJ, Schaper NC, on behalf of the International Working Group on the Diabetic Foot (IWGDF). The 2015 IWGDF guidance documents on prevention and management of foot problems in diabetes: development of an evidence-based global consensus: Development of the 2015 IWGDF Guidance. Diabetes Metab Res Rev. 2016 Jan;32:2-6.

[3] Chuan F, Tang K, Jiang P, Zhou B, He X. Reliability and Validity of the Perfusion, Extent, Depth, Infection and Sensation (PEDIS) Classification System and Score in Patients with Diabetic Foot Ulcer. Santanelli, di Pompeo d'Illasi F, editor. PLOS ONE. 2015 Apr 13;10(4):e0124739.

[4] Schaper NC. Diabetic foot ulcer classification system for research purposes: a progress report on criteria for including patients in research studies. Diabetes Metab Res Rev. 2004 May;20(S1):S90-5.

[5] Hüsers J, Hafer G, Heggemann J, Wiemeyer S, John SM, Hübner U. Predicting the amputation risk for patients with diabetic foot ulceration - a Bayesian decision support tool. BMC Med Inform Decis Mak. 2020 Aug 24;20(1):200.

[6] Pickwell K, Siersma V, Kars M, Apelqvist J, Bakker K, Edmonds M, et al. Predictors of lower-extremity amputation in patients with an infected diabetic foot ulcer. Diabetes Care. 2015 May;38(5):852-7.

[7] Ruopp MD, Perkins NJ, Whitcomb BW, Schisterman EF. Youden Index and Optimal Cut-Point Estimated from Observations Affected by a Lower Limit of Detection. Biom J Biom Z. 2008 Jun;50(3):419-30.

[8] Kruschke JK. Bayesian Assessment of Null Values Via Parameter Estimation and Model Comparison. Perspect Psychol Sci. 2011;6(3):299-312. 